\title{
Cocoa husks in diets of Italian heavy pigs
}

\author{
D. Magistrelli, ${ }^{1}$ L. Malagutti, G. Galassi, and F. Rosi
}

University of Milan, Department of Agricultural and Environmental Sciences, via G. Celoria, 2 - 20133 Milano, Italy

\begin{abstract}
The aim of the present study was to evaluate the effect of cocoa husks feeding on liver composition of the Italian heavy pig. Cocoa husks are byproducts derived from chocolate production and have a high content of proteins, lipids, and NDF. Cocoa husks are also rich in antioxidants, polyphenols in particular. Eight finishing pigs were divided into 2 groups: control group fed a traditional diet, based on cereals, and treatment group fed a diet obtained by substitution of $10 \%$ of the control diet with coarsely ground cocoa husks. The trial was conducted during the hot season and lasted $6 \mathrm{wk}$, at the end of which all the pigs were slaughtered. Cocoa husks diet reduced dry matter intake $(P<0.01)$ and energy intake $(P<0.01)$ but neither body
\end{abstract}

weight nor backfat thickness was affected by cocoa husks diet. Treatment did not influence carcass weight and hot dressing percentage but reduced liver weight $(P$ $<0.05)$, liver dry matter percentage $(P<0.01)$, DNA $(P$ $=0.01)$, and glycogen content $(P=0.01)$. By contrast, cocoa husks increased liver ether extract $(P=0.05)$ without affecting cholesterol content. Liver weight loss, reduction of protein synthesis, and a shift toward glycogen use instead of fat oxidation are considered metabolic strategies to reduce heat production under hot conditions. It is possible, therefore, that cocoa husks feeding promoted the process of acclimation because pigs needed less feeding to reach similar body and carcass weight as control pigs.

Key words: cocoa by-products, liver composition, pig, polyphenols.

$\begin{array}{rr}\text { (C) } 2012 \text { American Society of Animal Science. All rights reserved. } & \text { J. Anim. Sci. 2012.90:230-232 } \\ \text { doi:10.2527/jas53970 }\end{array}$

\section{INTRODUCTION}

Cocoa husks derived from the beans of the plant Theobroma cacao L., a plant tree originated in the rain forests of America, whose culture has extended to equatorial areas of Africa and Asia (Lecumberri et al., 2007). Cocoa beans are used worldwide for chocolate production and processing has the same basic steps: fermentation, roasting, and milling. At the end of the process, chocolate is destined for human consumption, but cocoa wastes, such as the husks, pulp, and by-products from cocoa butter, are commercially processed to produce a variety of products such as feedstuff, fertilizers, food products, and soap (McShea et al., 2008). Cocoa husks also have a high content of proteins, lipids, and antioxidants, polyphenols in particular (Lecumberri et al., 2007; Ramiro-Puig and Castell, 2009). According to several authors, cocoa polyphenols have several effects that may promote good health (Sanbongi et al., 1997; Matsui et al., 2005; McShea et al., 2008; Ramiro-Puig and Castell, 2009).

${ }^{1}$ Correspondence: damiano.magistrelli@guest.unimi.it
The use of dried cocoa pod husk in pig nutrition was tested by some authors (Oddoye et al., 2010). The present study aimed at estimating the effect of cocoa husks feeding on liver composition of finishing heavy pigs in order to evaluate if cocoa polyphenols consumption may influence the hepatic metabolism, which is strongly affected by environmental temperature.

\section{MATERIALS AND METHODS}

Eight castrated male pigs (Duroc $\times$ Large White) weighing $135 \pm 11.1 \mathrm{~kg}$ (mean $\pm \mathrm{SD}$ ) were divided into 2 homogeneous groups: control group (CTRL) fed a traditional pelleted diet, based on cereals, and a treatment group (COCOA) fed a diet obtained by substitution of $10 \%$ of the control diet with coarsely ground cocoa husks. The CTRL diet consisted of $15.0 \%$ of CP, $5.44 \%$ of minerals, $3.60 \%$ of ether extract (EE), $12.8 \%$ of $\mathrm{NDF}, 5.03 \% \mathrm{ADF}$, and $1.21 \%$ of $\mathrm{ADL}$; data are expressed as percentage of DM; GE, determined in an adiabatic bomb calorimeter (IKA Werke $\mathrm{GmbH}$ KG, Staufen, Germany), was $18.1 \mathrm{MJ} / \mathrm{kg}$. The CTRL 
diet was formulated using standard nutrient requirements of the Institut National de la Recherche Agronomique (Ferrando and Blum, 1989). The COCOA diet consisted of $15.4 \%$ of CP, $5.73 \%$ of minerals, $4.53 \%$ of EE, $14.2 \%$ of NDF, $7.19 \%$ ADF, and $2.22 \%$ of ADL; GE was 18.3 $\mathrm{MJ} / \mathrm{kg}$. The content of total polyphenols, determined using the Prussian Blue method (Budini et al., 1980), was 8.17 vs. $15.4 \mathrm{mg} / \mathrm{g}$ of DM for CTRL and COCOA diet, respectively. Pigs were fed twice daily and water was provided ad libitum. Animals were kept into individual cages in the same room under identical natural condition for light $(14.5 \pm 0.75 \mathrm{~h} / \mathrm{d})$, temperature $\left(27.1 \pm 2.49^{\circ} \mathrm{C}\right)$, and humidity $(65.0 \pm 4.65 \%)$. During the experimental period, individual DM intake was recorded daily; BW was recorded weekly.

After $6 \mathrm{wk}$, all the pigs were slaughtered after $18 \mathrm{~h}$ of fasting. All the animals used in the trial were reared for and slaughtered in accordance with European Union guidelines (Directive 86/609/CEE) and Italian legislation (DL 116, 1992 and DL 473, 1993). Body, carcass, and liver weights were recorded and hot dressing percentage was calculated. Backfat thickness was determined using the Fat-O-Meter and liver samples were taken and stored at $-80^{\circ} \mathrm{C}$ pending analysis. Liver samples were analyzed for DM by oven heating at $56^{\circ} \mathrm{C}$ for $72 \mathrm{~h}$. Liver DNA was determined according with Munro and Fleck (1966). After the determination of ether extract (AOAC International method 960.39), the extract was resuspended in $15 \mathrm{~mL}$ of n-exane and then analyzed for cholesterol using a commercial kit (Giesse Diagnostics s.r.l.). Glycogen was analyzed by the method of Kemp and Kits van Heijningen (1954) and soluble protein content was determined using a commercial kit (Pierce Biotechnology Inc.).

Data were analyzed by a 1-way ANOVA using the GLM procedure of SAS (SAS Institute Inc., Cary, NC) and are presented as mean $\pm \mathrm{SD}$. Differences between groups were considered significant for $P<0.05$.

\section{RESULTS AND DISCUSSION}

Cocoa husks diet reduced $(P<0.01)$ DM intake and energy intake but did not affect feed efficiency (G:F ratio was $0.31 \pm 0.03$ vs. $0.28 \pm 0.03 ; P=0.23$ ). Despite the differences in DM and energy intake, neither BW $(162 \pm 13.7 \mathrm{~kg}$ vs. $159 \pm 11.8 \mathrm{~kg} ; P=0.90)$ nor backfat thickness $(22.3 \pm 5.74 \mathrm{~mm}$ vs. $20.8 \pm 1.50 \mathrm{~mm} ; P=0.63)$ was affected by cocoa diet. Moreover, treatment did not influence carcass weight $(131 \pm 14.8$ vs. $129 \pm 11.9 \mathrm{~kg}$; $P=0.83)$ and dressing percentage $(80.5 \pm 2.32$ vs. $81.0 \pm$ $1.59 \% ; P=0.72)$.

As shown in Table 1, cocoa husks reduced liver weight, DM percentage, DNA, and glycogen content but increased ether extract without affecting liver cholesterol content. In growing pigs, the weights of internal organs, that is, digestive tract, liver, and kidneys, are positively correlated to endogenous heat production (Rinaldo et al., 2000). During the hot season, the weights of internal organs decrease in order to contribute to reduce endogenous heat production (Rinaldo et al., 2000). In growing pigs, weight loss is highest in the heart (Rinaldo et al., 2000) and the liver (van Milgen et al., 1998). The latter has been shown to be the major contributor to energy expenditure and heat production among the visceral organs (van Milgen et al., 1998). Moreover, Murase et al. (2008) observed that catechin consumption reduces liver weight in mice. It is possible, therefore, that in the present study, cocoa husks have enforced the effect of temperature on liver weight loss, as catechin, epicatechin, and their oligomers constitute the main polyphenols in cocoa (Lecumberri et al., 2007).

In humans, mice, and rats, catechin exerts its main effects by decreasing fatty acid synthesis and stimulating hepatic lipid catabolism, leading to reduced liver and abdominal fat (Matsui et al., 2005; Murase et al., 2008). Under hot conditions, such as those of the present experiment, the animal stores energy in the form of fat because fat deposition is a very efficient process, which contributes less to heat production (Webster, 1981). This leads to a shift toward the use of carbohydrates as energy substrate and a consequent increase in glucose output from the liver, as observed in the working horse exposed to high temperatures (Ott, 2005). Under hot conditions, protein synthesis could also be decreased in order to reduce endogenous heat production. Protein synthesis, in fact, is energetically expensive and largely contributes to total heat production (Webster, 1981). In the present study, cocoa husks feeding seemed to promote the process of coping with the hot conditions of the trial. This latter hypothesis is supported by the fact that treated pigs needed less feeding to reach similar body and carcass weight of the pigs fed the control diet.

Table 1. Weight and composition of liver of pigs fed a negative control diet (CTRL) and a 10\% cocoa husks diet (COCOA). Data are presented as mean \pm SD

\begin{tabular}{lccc}
\hline \hline & \multicolumn{2}{c}{ Treatment } & \\
\cline { 2 - 3 } Item & CTRL & COCOA & P-value \\
\hline Weight, g & $1893 \pm 47.2$ & $1634 \pm 177$ & 0.03 \\
Weight, \% BW & $1.17 \pm 0.12$ & $1.03 \pm 0.12$ & 0.14 \\
DM, \% & $30.5 \pm 1.37$ & $27.6 \pm 0.50$ & $<0.01$ \\
DNA, mg/g & $4.74 \pm 0.67$ & $3.20 \pm 0.40$ & 0.01 \\
DNA, mg/g DM & $15.4 \pm 2.55$ & $11.6 \pm 1.46$ & 0.05 \\
Ether extract, mg/g & $11.8 \pm 2.26$ & $15.1 \pm 1.53$ & 0.05 \\
Ether extract, mg/g DM & $38.6 \pm 6.13$ & $54.7 \pm 6.08$ & $<0.01$ \\
Cholesterol, mg/g & $2.13 \pm 0.60$ & $2.27 \pm 0.47$ & 0.73 \\
Cholesterol, mg/g DM & $7.35 \pm 2.24$ & $7.97 \pm 1.62$ & 0.67 \\
Glycogen, mg/g & $88.4 \pm 21.4$ & $24.6 \pm 1.99$ & 0.01 \\
Glycogen, mg/g DM & $289 \pm 60.7$ & $89.1 \pm 5.60$ & $<0.01$ \\
Soluble protein, mg/g & $84.8 \pm 8.85$ & $66.7 \pm 1.14$ & $<0.01$ \\
Soluble protein, mg/g DM & $279 \pm 39.3$ & $242 \pm 8.30$ & 0.11 \\
\hline
\end{tabular}


In conclusion, the present study suggests that under high temperatures as in this trial, the use of cocoa husks in the diet for finishing Italian heavy pigs decreases feed intake without depressing productive performances, as possible consequence of the effect of cocoa polyphenols in helping animal acclimation.

\section{LITERATURE CITED}

Budini, R., D. Tonelli, and S. Girotti. 1980. Analysis of total phenols using the Prussian Blue method. J. Agric. Food Chem. 28:12361238 .

Ferrando, R., and J. C. Blum. 1989. L'alimentation des animaux monogastriques: porc, lapin, volaille. 2nd ed. Institut National de la Recherche Agronomique, Paris, France.

Kemp, A., and A. J. M. Kits van Heijningen. 1954. A colorimetric micro-method for the determination of glycogen in tissues. Biochem. J. 56:646-648.

Lecumberri, E., R. Mateos, M. Izquierdo-Pulido, P. Ruperez, L. Goya, and L. Bravo. 2007. Dietary fibre composition, antioxidant capacity and physico-chemical properties of a fibre-rich product from cocoa (Theobroma cacao L.). Food Chem. 104:948-954.

Matsui, N., I. Ryoichi, E. Nishimura, M. Yoshikawa, M. Kato, M. Kamei, H. Shibata, I. Matsumoto, K. Abe, and S. Hashizume. 2005. Ingested cocoa can prevent high-fat diet-induced obesity by regulating the expression of genes for fatty acid metabolism. Nutrition 21:594-601.

McShea, A., E. Ramiro-Puig, S. B. Munro, G. Casadesus, M. Castell, and M. A. Smith. 2008. Clinical benefit and preservation of flavonols in dark chocolate manufacturing. Nutr. Rev. 66:630641.

Munro, H. N., and A. Fleck. 1966. The determination of nucleic acids. Methods Biochem. Anal. 14:113-176.

Murase, T., S. Haramizu, N. Ota, and T. Hase. 2008. Tea catechin ingestion combined with habitual exercise suppresses the agingassociated decline in physical performance in senescenceaccelerated mice. Am. J. Physiol. Regul. Integr. Comp. Physiol. 295:R281-R289.

Oddoye, E. O. K., S. W. A. Rhule, K. Agyente-Badu, V. Anchirinah, and F. Owusu Ansah. 2010. Fresh cocoa pod husk as an ingredient in the diets of growing pigs. Sci. Res. Essays 5:1141-1144.

Ott, E. A. 2005. Influence of temperature stress on the energy and protein metabolism and requirements of the working horse. Livest. Prod. Sci. 92:123-130.

Ramiro-Puig, E., and M. Castell. 2009. Cocoa: Antioxidant and immunomodulator. Br. J. Nutr. 101:931-940.

Rinaldo, D., J. Le Dividich, and J. Noblet. 2000. Adverse effects of tropical climate on voluntary feed intake and performance of growing pigs. Livest. Prod. Sci. 66:223-234.

Sanbongi, C., N. Suzuki, and T. Sakane. 1997. Polyphenols in chocolate, which have antioxidant activity, modulate immune functions in humans in vitro. Cell. Immunol. 177:129-136.

van Milgen, J., J. F. Bernier, Y. Lecozler, S. Dubois, and J. Noblet. 1998. Major determinants of fasting heat production and energetic cost of activity in growing pigs of different body weight and breed/ castration combination. Br. J. Nutr. 79:509-517.

Webster, A. J. F. 1981. The energetic efficiency of metabolism. Proc. Nutr. Soc. 40:121-128. 